\title{
Dynamical Properties of AIN Nanostructures and Heterogeneous Interfaces Predicted using COMB Potentials
}

Kamal Choudhary ${ }^{1}$, Tao Liang ${ }^{1,2}$, Kiran Mathew $^{3}$, Benjamin Revard ${ }^{3}$, Aleksandr Chernatynskiy $^{1,4}$, Simon R. Phillpot ${ }^{1}$, Richard G. Hennig ${ }^{1,3}$ and Susan B. Sinnott ${ }^{1,2}$,

${ }^{1}$ Department of Materials Science and Engineering, University of Florida, Gainesville, FL, 32611

${ }^{2}$ Department of Materials Science and Engineering, Pennsylvania State University, University Park, PA 16801

${ }^{3}$ Department of Materials Science and Engineering, Cornell University, Ithaca, New York, 14853

${ }^{4}$ Department of Physics, Missouri University of Science \& Technology, Rolla, Missouri, 65409

\begin{abstract}
A new empirical variable charge potential has been developed for AlN within the third-generation charge optimized many-body (COMB3) potential framework. The potential is able to reproduce the fundamental physical properties of AlN, including cohesive energy, elastic constants, defect formation energies, surface energies and phonon properties of AlN obtained from experiments and first-principles calculations. The thermodynamic properties of the $\mathrm{Al}(111)-\mathrm{AlN}(10 \overline{1} 0)$ and $\mathrm{Al}_{2} \mathrm{O}_{3}(0001)-\mathrm{AlN}(10 \overline{1} 0)$ interfaces and the tensile response of $\mathrm{AlN}$ nanowires and nanotubes are investigated in classical molecular dynamical (MD) simulations using this COMB3 potential. The results demonstrate that the potential is well suited to model heterogeneous materials in the Al-O-N system. Most importantly, the fully transferrable potential parameters can be seamlessly coupled with existing COMB3 parameters of other elements to enable MD simulations for an even wider range of heterogeneous materials systems.
\end{abstract}

Keywords: Aluminum nitride, COMB3, nanowires, nanotubes, interface.

*Corresponding author. Email: sinnott@ matse.psu.edu 


\section{Introduction}

Aluminum nitride (AIN) is an important III-V semiconductor material due to its wide band gap, high thermal conductivity, low thermal expansion, and non-toxicity [1]. Nanostructures of AlN are used for field emitters in flat panel displays [2] and photo-detectors [3, 4]. Additionally, thin films of AlN and aluminum oxynitride are important engineering protective coatings [5-9]. Recently Cremer et al. [10] demonstrated experimentally that Al-O-N coatings are better diffusion barriers for components under high thermal load, e.g. in gas turbines, than pure $\mathrm{Al}_{2} \mathrm{O}_{3}$. Improved understanding of the thermodynamic and mechanical phenomena that take place in these systems at all levels, including the atomic scale, can play a critical role in optimizing their performance $[11,12]$.

The Al-O-N system has been the focus of several computational studies using densityfunctional theory (DFT) as well as molecular dynamics (MD) simulations with empirical potentials. For example, Felice and Northrup [13] used DFT calculations to explore the effect of the chemical potential of $\mathrm{Al}$ on the formation energies and structures of $\mathrm{AlN}$ on $\mathrm{Al}_{2} \mathrm{O}_{3}$. In addition, Ogata et al. [14] used DFT calculations to compare the mechanical properties of $\mathrm{Al} / \mathrm{AlN}$ heterostructures to those of $\mathrm{Al}$ and $\mathrm{AlN}$. They found that the failure of the Al/AlN interface occurs at the $\mathrm{Al}$ layer of the interface. The strength predicted from the DFT calculations was about two times less than that predicted from conventional Griffith's theory[15] for materials. More recently, Li et al. [16] used DFT to examine atomic and molecular hydrogen storage in AlN nanowires and nanotubes. They found atomic hydrogen attaches more favorably

to $\mathrm{N}$-sites than Al-sites on the nanowire surfaces. While DFT is a compelling high-fidelity quantum mechanical approach, it is computationally intensive and is usually restricted to the study of small systems. 
Classical MD simulations may be applied to larger systems, but require empirical potentials to determine the forces on the atoms. Various types of empirical potentials exist, including fixed charge, dynamic charge, non-reactive and reactive. With regards to AlN, a Buckingham-type of fixed charge potential was developed by Chisholm et al. [17] which predicted reasonable lattice constants, elastic constants and defect formation energies. Several Tersoff-based reactive bondorder interatomic potentials have been parameterized for AlN [18-21] and successfully applied to examine its thermal expansion [18] and the properties of AlN nanotubes [22]. Vashishtha et al. [23] proposed a potential for AlN with three-body interaction terms along the lines of the Stillinger-Weber potential and used it to investigate the mechanical and thermal properties of crystalline and amorphous AlN. The potential did well in predicting the lattice constant, elastic constant, and vibrational density of states using fixed charges on the atoms. In order to capture important aspects of heterogeneous Al-O-N systems such as AlN coatings deposited on oxidized Al surfaces, a potential that is both reactive and has dynamically varying charge is highly desirable. Here, we report on the development of a third-generation, charge optimized manybody (COMB3) reactive interatomic potential for the AlN system that is fully compatible with existing COMB3 potentials for $\mathrm{Al}$ [24], $\mathrm{Al}_{2} \mathrm{O}_{3}$ [25] and $\mathrm{O}-\mathrm{N}$ [26]. We further demonstrate its capabilities by using it in MD simulations to examine the properties of bulk AlN, the thermodynamic stability of $\mathrm{Al} / \mathrm{Al}_{2} \mathrm{O}_{3} / \mathrm{AlN}$ interfaces, and the mechanical responses of $\mathrm{AlN}$ nanowires and nanotubes. Similar COMB3 potentials have been developed for $\mathrm{Cu}$ [27], U [28], $\mathrm{Zr}$ [28], Ti [29], some metal oxides [25, 30, 31], and metal nitrides [32].

The paper is organized as follows: first, a short description of the COMB3 potential and details of its parameterization and testing are given in Section II. In Section III, the results of the 
MD simulations performed using the COMB3 potential are presented and discussed. Finally, the main findings of the work are summarized in Section IV.

\section{COMB3 Potential Development}

The COMB3 potential is a reactive and dynamically variable charge potential. The reader is referred to Ref. [32] for fill details of the formalism. Briefly, the total potential energy, $U^{\text {tot }}$, is expressed as:

$$
U^{\text {tot }}(r, q)=U^{e s}(q, r)+U^{\text {short }}(q, r)+U^{v d W}(r)+U^{\text {corr }}(r)
$$

where $U^{e s}$ represents the electrostatic potential, $U^{\text {short }}$ the short range energy terms, $U^{v d W}$ the van der Waals interactions, and $U^{\text {corr }}$ is a correction term. Each term has a complex form with disposable parameters that are determined to give the best fit to a database of experimental and DFT values of salient physical properties.

\subsection{Parameterization}

Twelve structures were considered during the parameter fitting of the AlN COMB3 potential, namely: wurtzite AlN (w-AlN) (space group P6 $\underline{3} \underline{\text { mc }}$ ), zinc blende (space group $F \overline{4} 3 m$ ), rock-salt (space group $F m \overline{3} m$ ), calcium fluorite $\left(\mathrm{CaF}_{2}\right.$ structure, space group $\left.F m \overline{3} m\right)$, cesium chloride (space group $P m 3 m$ ), point defects (vacancies and interstitials including $\mathrm{V}_{\mathrm{Al}}, \mathrm{V}_{\mathrm{N}}, \mathrm{Al}_{\mathrm{i}}$, and $\mathrm{N}$ in w-AlN), and the surface energy of the w-AlN (10 $\overline{1} 0)$ non-polar surface. The w-AlN structure was taken in the orthorhombic, rather than rhombohedral representation to simplify the calculations. The solid-state phases with different stoichiometries in the fitting database were 
included to enable the parameterization of AlN systems as a function of the number and angular orientation of their neighbors.

The properties of these structures were determined using DFT with the PBE (Perdew, Burke, and Ernzerhof)-GGA (generalized gradient approximation) density functional using the Vienna Ab initio Simulation Package (VASP) software package [33-36]. The calculations used a $500 \mathrm{eV}$ energy cutoff; the self-consistency energy convergence criterion was taken as $10^{-6} \mathrm{eV}$ with a $10^{-2}$ $\mathrm{eV} / \AA$ force convergence criterion. The target values for the lattice constant, elastic constants, heats of formation, point defect energies, surface energies, elastic constants and bulk modulus of w-AlN were used in the fitting database from the DFT calculations, as indicated in Table. 1. Here, lattice constants and heats of formation data were taken from experimental measurements, while other data were taken from DFT calculated values.

It is important to calibrate the relative stability of the materials during the fitting procedure. We used the heat of formation and heat of reaction as the thermodynamic quantities to compare the relative stability of the structures. The heat of formation is the energy required to form the material from its constitutive elements, which were chosen to be face-centered cubic (FCC) Al and an isolated, gas-phase $\mathrm{N}_{2}$ molecule, respectively,

$$
x A l+y \frac{1}{2} N_{2} \rightarrow A l_{x} N_{y}, \Delta H_{f}=(x+y) E_{\text {atom }}-x \mu_{A l}-y \mu_{\frac{1}{2} N_{2}}
$$

where $x$ and $y$ are stoichiometric coefficients, $\Delta H_{f}$ is the heat of formation and $\mu$ is the chemical potential. After the heat of formation for each individual compound was calculated, the heats of reactions were determined as the energy difference between phases, such as, for example, between the bulk w-AlN phase (A) and a different phase (B): 
$m A l+b N(A) \rightarrow x A l+y N(B)$

$\Delta H_{r x n}=(m+n) E_{\text {atom }(A)}-(x+y) E_{\text {aton }(B)}-(x-m) \mu_{A l}-(y-n) \mu_{\frac{1}{2} N_{2}}$

In Eq. (4), $x, y, m$ and $n$ are stoichiometric coefficients, $\Delta H_{r x n}$ is the heat of reaction, $E_{\text {atom }}$ is the energy per atom of a system and $\mu$ is the chemical potential. In order for the w-AlN phase to be the ground state, the heats of reaction for other phases should be positive.

Optimization of COMB3 parameters was carried out using the POSMat (Potential Optimization Software for Materials) [37] software, which employs a simplex algorithm [38]. The optimization uses the weighted least squares method for absolute deviation of lattice parameters, elastic constants, surface energies and defect formation energies. The parameters were confined by keeping the elastic constant, bulk modulus and other properties mentioned above to be within $20 \%$ of the target values. The COMB3 results and the parameterization target values are given in Table 1; the corresponding parameters developed for the AlN COMB3 potential are distributed in LAMMPS open source MD software [39] and presented in the Appendix. 
Table 1. Properties of w-AlN predicted by the indicated computational methods and/or measured by experimental methods. The point defect energies and heats of reactions are given in units of eV.

\begin{tabular}{|c|c|c|c|c|c|}
\hline$\underline{\mathrm{AlN}}$ & Exp./ DFT & $\begin{array}{l}\text { Chisholm et } \\
\text { al. [17] }\end{array}$ & $\begin{array}{l}\text { Vashishtha } \\
\text { et al. [23] }\end{array}$ & $\begin{array}{l}\text { Tersoff } \\
{[21]}\end{array}$ & COMB3 \\
\hline $\mathrm{a}(\mathrm{nm})$ & $0.311^{\mathrm{a}}$ & 0.311 & - & 0.311 & 0.311 \\
\hline $\mathrm{c} / \mathrm{a}$ & $1.601^{\mathrm{a}}$ & 1.60 & - & 1.63 & 1.62 \\
\hline$\Delta \mathrm{H}_{\mathrm{f}}(\mathrm{eV} /$ atom $)$ & $-1.56^{\mathrm{a}}$ & & & -1.64 & -1.50 \\
\hline B (GPa) & $228^{\mathrm{b}}$ & 248 & 211 & 210 & 218 \\
\hline $\mathrm{C}_{11}(\mathrm{GPa})$ & $464^{b}$ & 417 & 435 & 392 & 463 \\
\hline $\mathrm{C}_{12}(\mathrm{GPa})$ & $149^{\mathrm{b}}$ & 178 & 148 & 137 & 92 \\
\hline $\mathrm{C}_{13}(\mathrm{GPa})$ & $116^{\mathrm{b}}$ & 152 & 107 & 101 & 104 \\
\hline $\mathrm{C}_{33}(\mathrm{GPa})$ & $409^{b}$ & 432 & 356 & 428 & 437 \\
\hline $\mathrm{C}_{44}(\mathrm{GPa})$ & $128^{\mathrm{b}}$ & 125 & 81 & 114 & 194 \\
\hline$\Delta \mathrm{H}_{\mathrm{rxn}}(\mathrm{ZB}-\mathrm{WZ})$ & 0.05 & & & & 0.25 \\
\hline$\Delta \mathrm{H}_{\mathrm{rxn}}(\mathrm{NaCl}-\mathrm{WZ})$ & 0.4 & & & & 2.2 \\
\hline$\Delta \mathrm{H}_{\mathrm{rxn}}(\mathrm{CsCl}-\mathrm{WZ})$ & 4.03 & & & & 1.37 \\
\hline$\Delta \mathrm{H}_{\mathrm{rxn}}\left(\mathrm{CaF}_{2}-\mathrm{WZ}\right)$ & 4.64 & & & & 2.24 \\
\hline $\begin{array}{l}\Delta \mathrm{H}_{\mathrm{rxn}} \quad\left(P 6_{3} / m m c-\right. \\
\mathrm{WZ})\end{array}$ & 0.27 & & & & 1.21 \\
\hline $\mathrm{N}$ vacancy & $\begin{array}{l}6.79,1.44- \\
5.36^{\mathrm{c}}\end{array}$ & & & & 4.8 \\
\hline Al vacancy & $\begin{array}{l}7.97,2.36- \\
6.31^{\mathrm{c}}\end{array}$ & & & & 0.30 \\
\hline $\mathrm{N}$ interstitial & 3.81 & & & & 8.92 \\
\hline $\mathrm{Al}$ interstitial & 11.1 & & & & 12.8 \\
\hline$\gamma(10 \overline{1} 0)\left(\mathrm{J} / \mathrm{m}^{2}\right)$ & $2.3^{\mathrm{d}}$ & & & & 2.13 \\
\hline
\end{tabular}

Ref. a [40], Ref. b [41], Ref. c [42], Ref. d [43] 


\section{Results and Discussion}

\subsection{Bulk Properties}

The bulk and surface properties of AlN predicted by COMB3 are provided in Table 1 along with comparisons to experimental data, the results of DFT calculations, as well as to published data obtained using the Tersoff potential [21], the potential of Vashishtha et al. [23] and that of Chisholm et al. [17]. The heats of reaction for all the phases considered besides Wurtzite (w)AlN were predicted to be positive by COMB3, which means that it correctly predicts the w-AlN phase to be the lowest energy state amongst the structures considered here. In addition, the average charges on the $\mathrm{Al}$ and $\mathrm{N}$ in bulk w-AlN are $+0.32 \mathrm{e}$ and $-0.32 \mathrm{e}$, respectively. These values are comparably smaller in magnitude than the DFT based Bader charge analysis of $\pm 1.85 \mathrm{e}$ [44] and the $\pm 1.1 \mathrm{e}$, which was also used by the potential of Vashishta and co-workers.

Table 1 shows the defect formation energy for interstitials and vacancies that are determined using

$$
E^{f}=E^{\text {def }}-E^{p e r f} \pm \sum_{i} n_{i} \mu_{i},
$$

where $E_{d e f}$ and $E_{p e r f}$ are defective and perfect structure energies of w-AlN, respectively, $\mu_{i}$ is the chemical potential of the $\mathrm{i}^{\text {th }}$ atom that is removed or added, and $n_{i}$ is the number of defects. Although defect formation energies were included in the fitting, concessions had to be made in order for the potential to predict good values for both the heats of formation and the elastic constants. Consequently, the $\mathrm{Al}$ vacancy and $\mathrm{N}$ interstitial defect formation energy values were underestimated by COMB3 compared to the DFT target values, but the non-negative nature of the defect formation energies was an important outcome that ensures phase stability. 
In addition to the defect formation energies, the parameters were further refined through fitting to the non-polar surface energy of AlN $(10 \overline{1} 0)$. The slab thickness was taken as $1.45 \mathrm{~nm}$ in the case of DFT and $2.50 \mathrm{~nm}$ in the case of COMB3. To simulate the surfaces, $1.50 \mathrm{~nm}$ of vacuum was added in the direction normal to the $(10 \overline{1} 0)$ surface in the case of DFT and $3.00 \mathrm{~nm}$ in the case of COMB3 calculations. The surface energies were calculated as:

$$
\sigma=\frac{E_{\text {slab }}-E_{\text {bulk }}}{2 A}
$$

where $E_{\text {slab }}$ is the total energy of the slab, $E_{\text {bulk }}$ is the energy of the bulk structure, and $A$ is the surface area of the $(10 \overline{1} 0)$ surface. The surface energy was found to be comparable to the DFT values.

The phase order for the Al-N systems considered here from DFT was w-AlN<zincblend $<\mathrm{P}_{3} / \mathrm{mmc}<[\mathrm{NaCl}]<[\mathrm{CsCl}]<\left[\mathrm{CaF}_{2}\right]$, while that from $\mathrm{COMB} 3$ was $w$-AlN $<$ zincblend $<\mathrm{P}_{3} / \mathrm{mmc}<<[\mathrm{CsCl}]<[\mathrm{NaCl}]<\left[\mathrm{CaF}_{2}\right]$. As the fitting database consisted of a limited number of structures, it was important to investigate the performance of the potential using a random structure search genetic algorithm. This is a very stringent test for a classical potential, and its negative results should not be viewed as disqualifying this particular potential for use, but rather as a limiting factor for its applications. In particular, the genetic algorithm in the GASP software package [45] was employed to map the energy landscapes of the $\mathrm{AlN}_{2} \mathrm{Al}_{2} \mathrm{O}_{3}$ and $\mathrm{Al}-\mathrm{O}-\mathrm{N}$ COMB3 potentials by searching for possible outlying low energy structures. Specifically, the genetic algorithm searched for low energy structures and their fitness were calibrated based on the energies of the structures obtained from the COMB3 potential. 
The structures used in the fitting database were considered to be initial guesses in the parent generation of the algorithm. New structures were then constructed using the genetic algorithm through mutation and mating operations on the parent structures. After subsequent generations of the convex hull plots were illustrated in Fig. 1. The algorithm was constrained within some hard parameters: maximum and minimum lattice parameters, minimum bond-lengths of $\mathrm{Al}-\mathrm{O}$ and $\mathrm{Al}-$ $\mathrm{N}$ system (about $80 \%$ of the equilibrium bond length), and the maximum number of structures in each generation, which is a typical setting for GA [46]. Hence, a total of 1200 structures were considered and their energies were determined using COMB3. Only the structures with negative energies were subsequently plotted in the convex hull plot. The primitive structures were replicated in a supercell of at least $21 \AA$ size to meet long range Coulomb cut off criteria of COMB3.

The structures lying on the convex hull plot are stable. The stable structures are shown as blue points while the unstable ones are shown as red points on the convex hull plot. Any other structure not lying on the curve can be represented as a mixture of the stable structures. In the case of the Al-O plot, $\alpha-\mathrm{Al}_{2} \mathrm{O}_{3}$ was found on the curve. The rock-salt $\mathrm{AlO}$ structure was also found in the convex hull plot. Experimentally or computationally (using DFT Materials Project computed data [47]), $\mathrm{AlO}$ is not found in the actual phase diagram of Al-O systems. It is to be noted that the results do not indicate that the rock-salt phase is more stable than $\alpha-\mathrm{Al}_{2} \mathrm{O}_{3}$. Rather, it indicates that at a 50\%-50\% ratio of of $\mathrm{Al}$ and $\mathrm{O}, \mathrm{AlO}$ is predicted to form and at a $60 \%-40 \%$ ratio of $\mathrm{Al}$ and $\mathrm{O}, \alpha-\mathrm{Al}_{2} \mathrm{O}_{3}$ is predicted to form.

In the case of the Al-N search, the AlN phase (with composition fraction 0.5 in Fig. 1b) was predicted to be $\sim 0.05 \mathrm{eV}$ energy/formula unit above the convex hull curve. Additionally, $\mathrm{AlN}_{4}$ was found to be a stable phase in the Al-N phase diagram search. Similar to the Al-O case, AlN 4 
is not a stable structure in either the experimental or the first-principles computational phase diagrams of the Al-N system. As a point of comparison, the prediction of non-existent phases to be stable on convex hull plots was also observed during ReaxFF parametrization of a $\mathrm{LiS}_{2}$ potential [48].

In addition to the $\mathrm{Al}-\mathrm{O}$ and $\mathrm{Al}-\mathrm{N}$ systems, we also generated convex hull plot for the $\mathrm{Al}-\mathrm{O}-\mathrm{N}$ ternary system and the results are illustrated in Fig. 1c. Some of the Al-O-N structures that were predicted to be stable were $\mathrm{Al}_{3} \mathrm{O}_{3} \mathrm{~N}, \mathrm{AlO}_{4} \mathrm{~N}, \mathrm{AlO}_{3} \mathrm{~N}_{3}, \mathrm{AlO}_{2} \mathrm{~N}_{8}, \mathrm{AlON}_{7}, \mathrm{AlON}_{10}, \mathrm{Al}_{2} \mathrm{ON}_{7}$ and $\mathrm{Al}_{5} \mathrm{ON}_{5}$. It is to be noted here that Al-O-N ternary fitting was not carried out separately. In all cases the incorrect predictions of the genetic algorithm search may be considered to be artifacts of the limited fitting database in our parametrization procedure and demonstrate the scope of further improvement of the parameters. However, the current parametrization is predicted to perform well for compositions in the vicinity of the fitted database structures. 


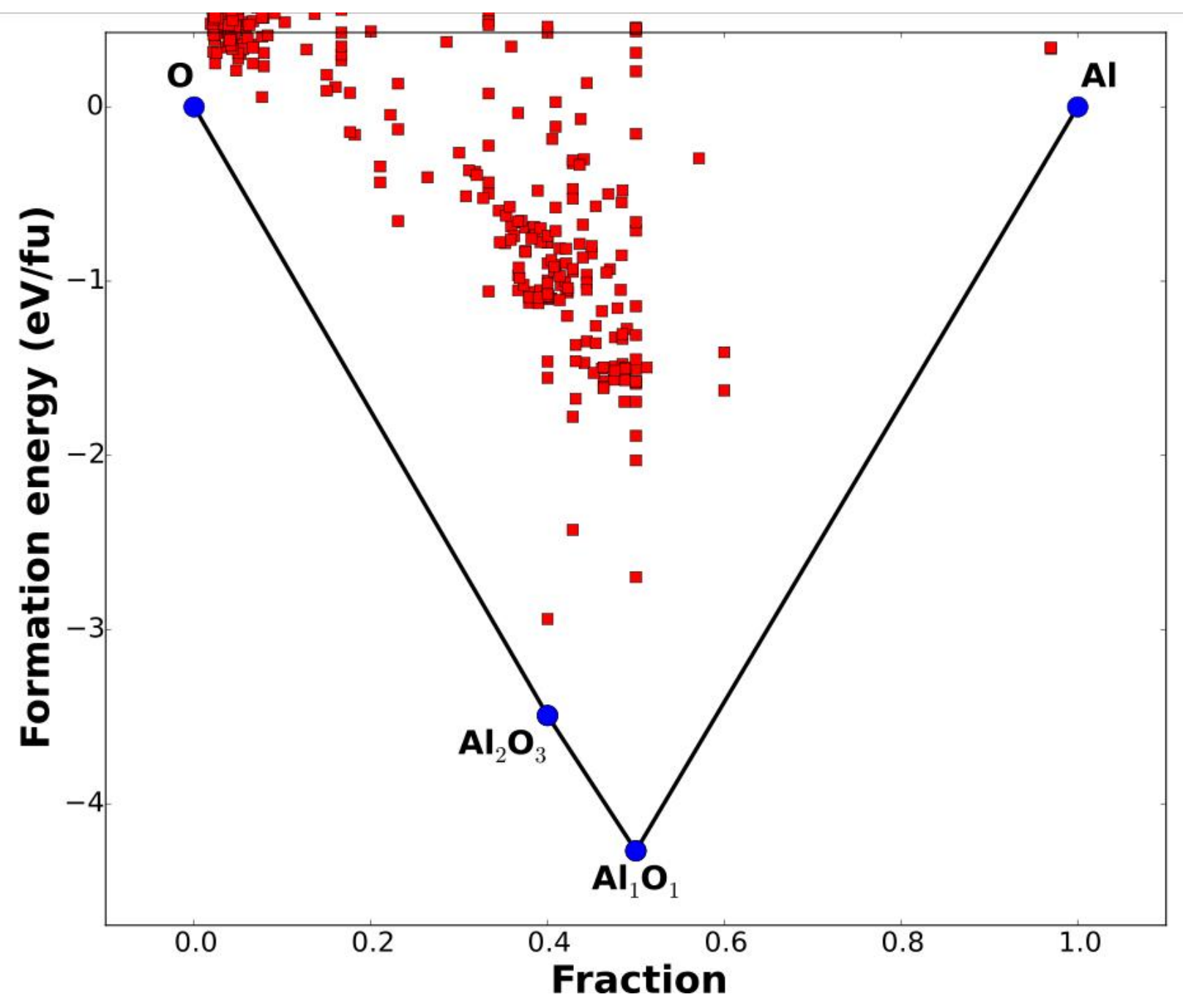

Fig. 1a Genetic algorithm search results for COMB3 Al-O. 


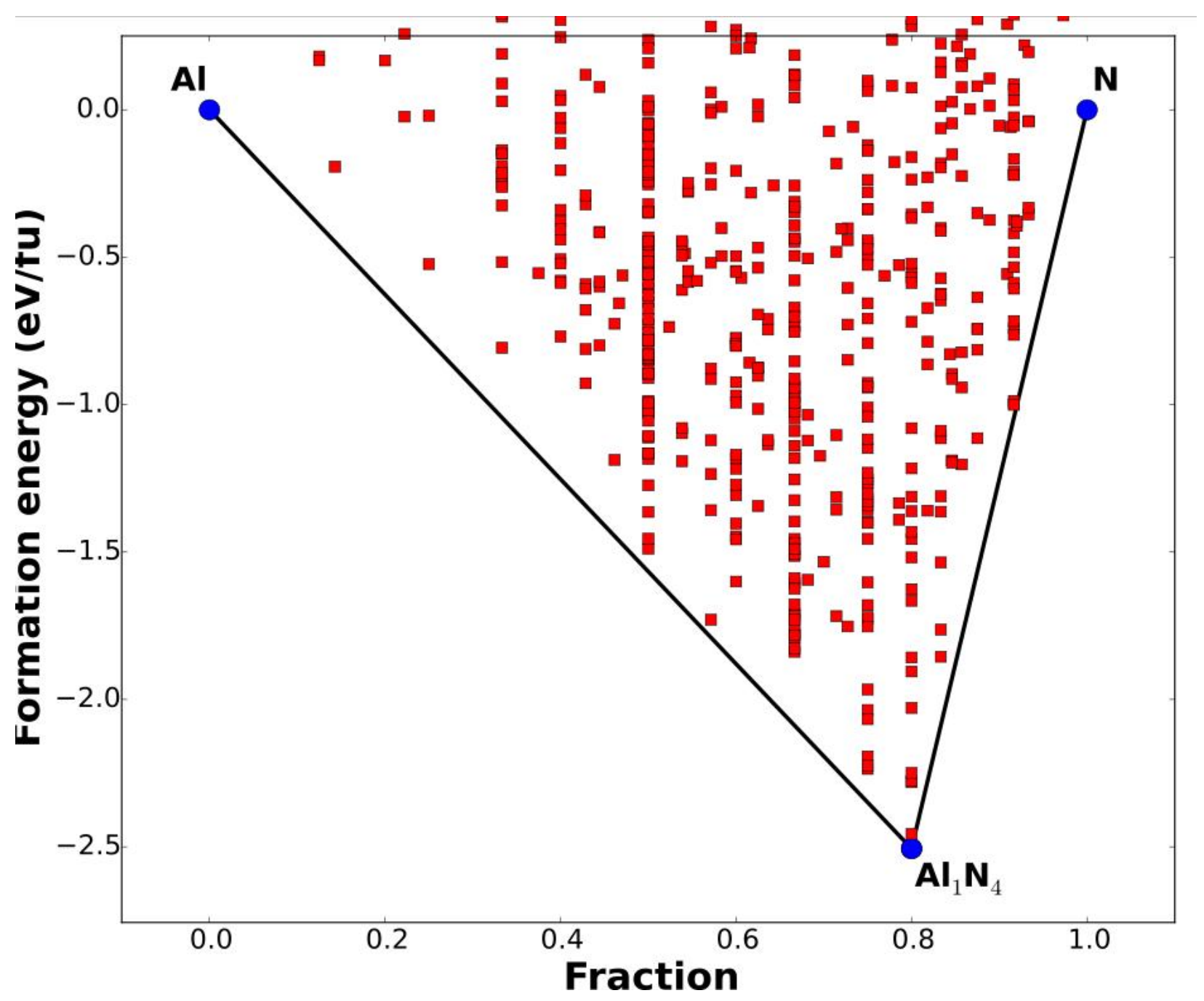

Fig. 1b Genetic algorithm search results for COMB3 Al-N. 


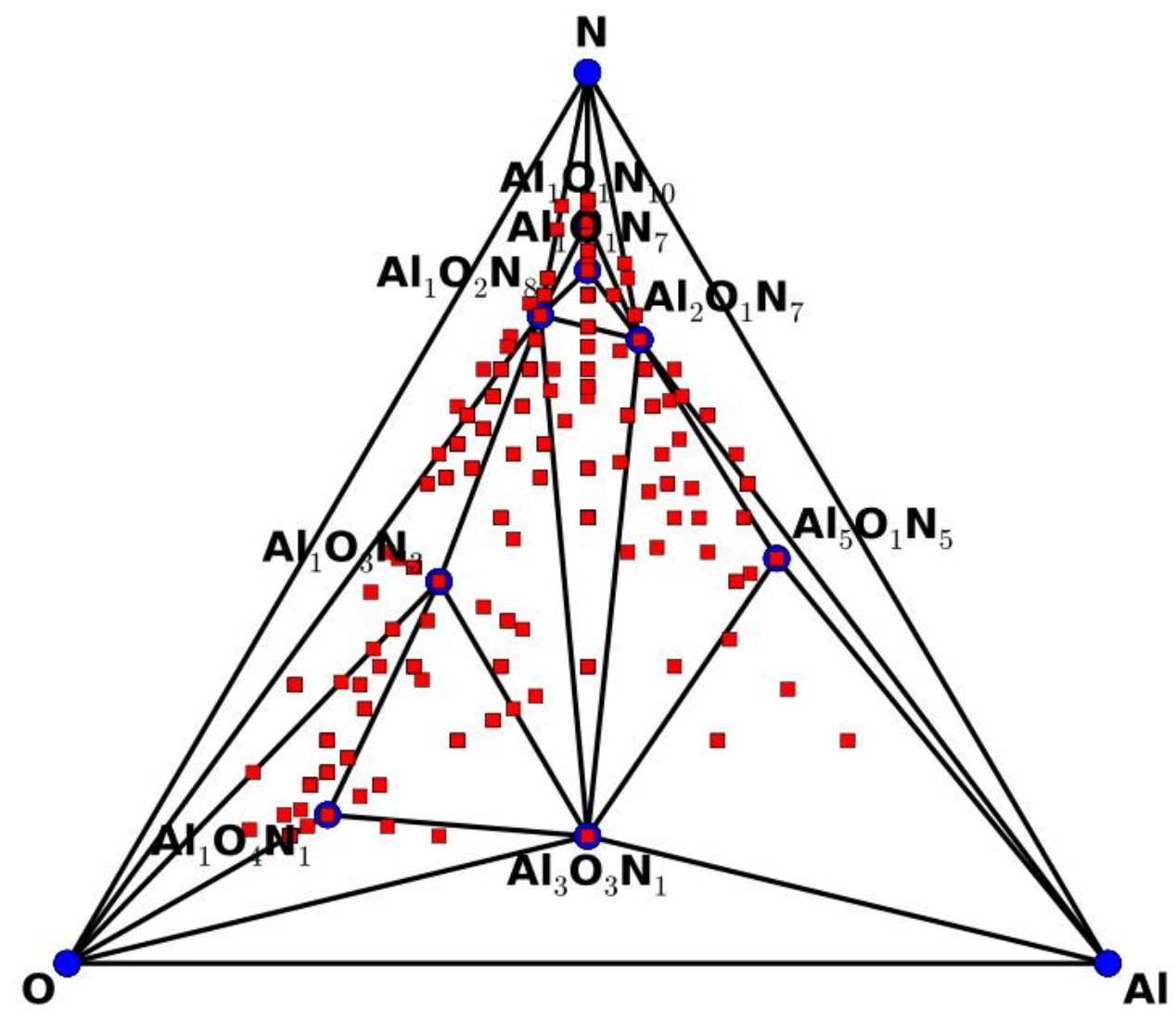

Fig. 1c Genetic algorithm search results for COMB3 Al-O-N.

Taken as a whole, COMB3 qualitatively predicts phase stability, lattice constants, elastic constants, defect formation energies and surface energies for w-AlN reasonably well. Quantitative agreement with known structure-property values is also generally good, but various underestimations in defect formation energies and deviations from target DFT surface energies and the true phase diagram do not allow perfect quantitative agreement for all properties. 


\subsection{Phonon Density of States}

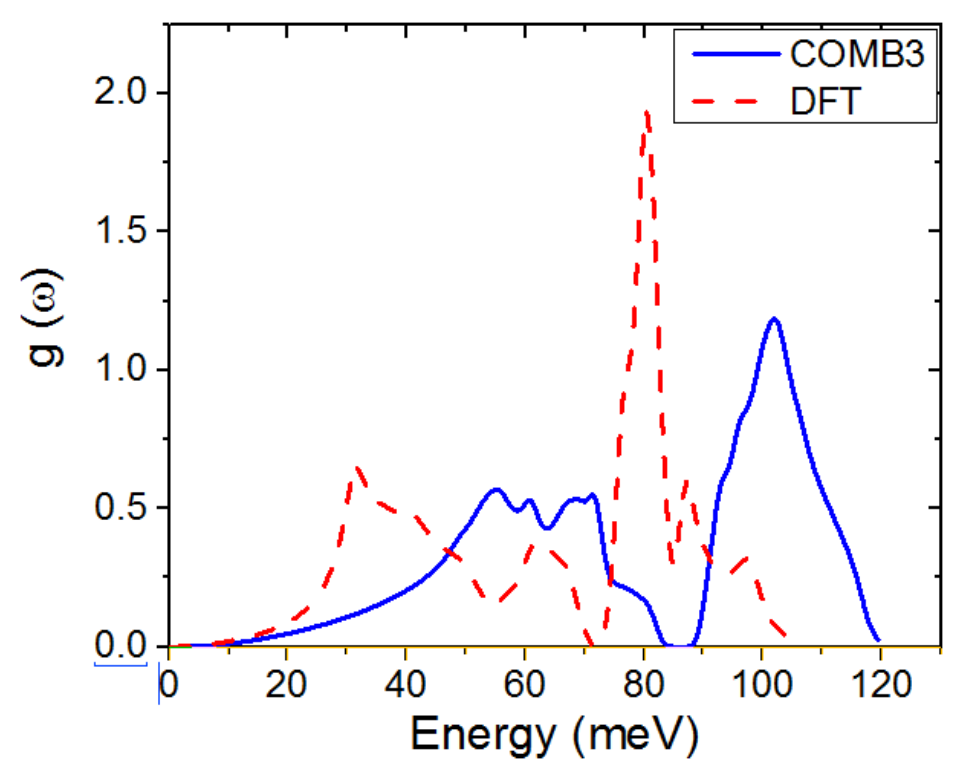

Fig. 2 Phonon density of states for w-AlN calculated using DFT and the COMB3 potential.

The phonon density of states (PDOS) [49, 50] for w-AlN was determined to test the dynamical stability of the AIN COMB3 potential. In particular, the predicted PDOS from DFT and COMB3 were obtained using the PhonTS software [51]. A k-point mesh of 35x35x35 was used for the PDOS calculations for both methods. The second derivative of energies were calculated using the finite difference method with a step size of $0.05 \AA$. The overall features of the PDOS are the same in both cases, as indicated in Fig. 2. However, the Debye like $E^{2}$ peak was predicted to be below $30 \mathrm{meV}$ using DFT while for COMB3 it extends up to $50 \mathrm{meV}$. The differences in the COMB3 and DFT results are likely due to the fact that we did not explicitly fit to these perturbed structures. However, the absence of imaginary phonon frequencies signifies that the w-AlN structure is predicted to be dynamically stable, which is an key property for the COMB3 potential to properly capture.

\section{3 $\mathrm{Al}-\mathrm{Al}_{2} \mathrm{O}_{3}$-AIN Interfaces}


To further test the reliability of the COMB3 potential, the work of adhesion of the $\mathrm{Al}$ (111)$\operatorname{AlN}(10 \overline{1} 0)$ and $\mathrm{AlN}(10 \overline{1} 0)-\alpha-\mathrm{Al}_{2} \mathrm{O}_{3}(0001)$ interfaces were investigated as they are of major technical importance [52]; these interface structures are illustrated in Fig. 3. The interface building algorithm by Zur et al. [53] was used to construct these heterogeneous interfaces with a minimal amount of mismatch. The algorithm was designed to search for all possible surface matches during the construction of the interface.

The $\operatorname{Al}(111)-\operatorname{AlN}(10 \overline{1} 0)$ contained 64800 atoms in $\mathrm{Al}$ and 58320 atoms in $\mathrm{AlN}$, respectively. The mismatch between the two surfaces was 0.04 . The strain to achieve matching periodic boundary conditions within the plane of the interface was applied to the Al layer. The energies of the structures were minimized with the conjugate gradient algorithm. We calculated the work of adhesion for $\operatorname{Al}(111)-\operatorname{AlN}(10 \overline{1} 0)$ to be $2.30 \mathrm{~J} / \mathrm{m}^{2}$ using eq. 7 :

$W_{\text {adh }}=\left(E_{\text {slab-1 }}^{\text {Tot }}+E_{\text {Slab-2 }}^{\text {Tot }}-E_{\text {lnff }}^{\text {Tot }}\right) / A$

where $W_{\text {adh }}, E_{\text {Intf }}^{\text {Tot }}, E_{\text {Slab-1 }}^{T o t}, E_{\text {Slab-2 }}^{\text {Tot }}, A$ are the work of adhesion for the interface, energies of slab-1 and slab-2, and area of the interface, respectively. The work of adhesion for this system from our DFT calculation is $2.66 \mathrm{~J} / \mathrm{m}^{2}$. The Al-N equilibrium bond length was found to be $0.25 \mathrm{~nm}$ at the interface. As the $\mathrm{Al}$ atoms near the AlN surface acquire a positive charge $(0.05 \mathrm{e})$, partial ionic charge was predicted at the interface $\mathrm{Al}$ atoms. The work of adhesion value indicates that the AlN COMB3 potential is in good agreement with the first-principles calculation data.

Next, we calculated the work of adhesion for the $\mathrm{Al}_{2} \mathrm{O}_{3}$ (0001)-AlN (10 $\left.\overline{1} 0\right)$ system consisting of 66000 atoms in $\mathrm{Al}_{2} \mathrm{O}_{3}$ and 28000 atoms in AlN. As the system consisted of dissimilar ceramic materials, mismatch was constrained to within 0.01 . It is to be noted that for 
the present system DFT calculations are too computationally expensive and were thus not carried out. The work of adhesion was determined to be $0.55 \mathrm{~J} / \mathrm{m}^{2}$ which is much less than that of the $\operatorname{Al}(111)-\operatorname{AlN}(10 \overline{1} 0)$ interface. This result indicates that the $\operatorname{Al}(111)-\operatorname{AlN}(10 \overline{1} 0)$ interface should be more thermodynamically stable than the $\mathrm{Al}_{2} \mathrm{O}_{3}(0001)$ - $\mathrm{AlN}(10 \overline{1} 0)$ interface. The Al-N bond length found in the case of $\mathrm{Al}_{2} \mathrm{O}_{3}$-AlN interface case was $0.26 \mathrm{~nm}$. In addition, the correct trends have already been predicted for the $\mathrm{Al} / \mathrm{Al}_{2} \mathrm{O}_{3}$ systems using COMB3 compared to DFT and experimental results [25]. Hence, the results $\mathrm{Al} / \mathrm{Al}_{2} \mathrm{O}_{3} / \mathrm{AlN}$ COMB potential can be utilized for further in-depth study of these technologically important interface systems. 


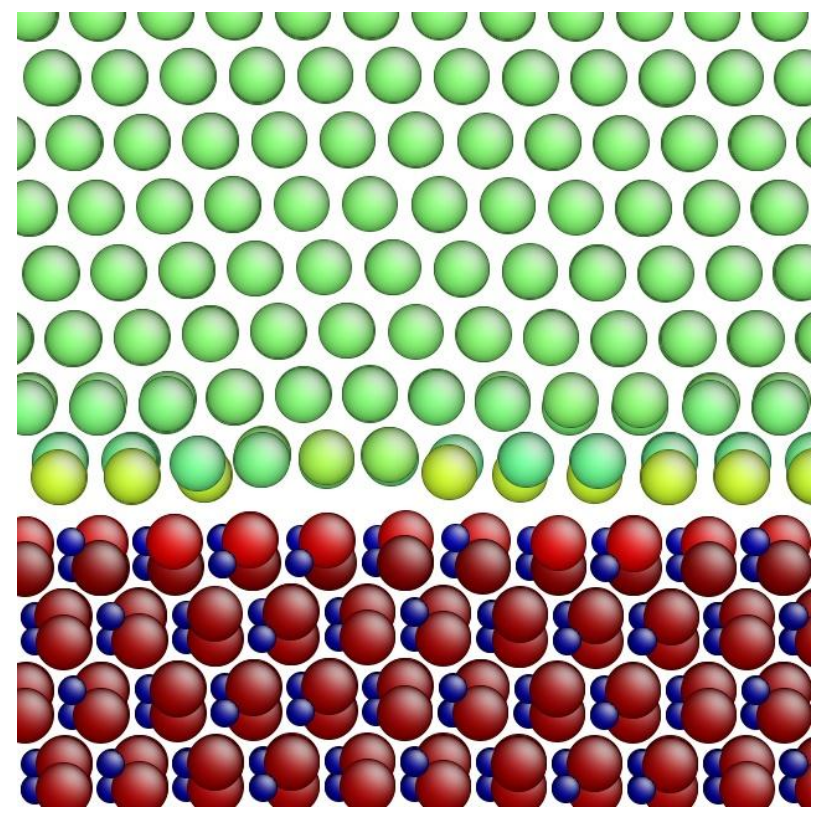

A

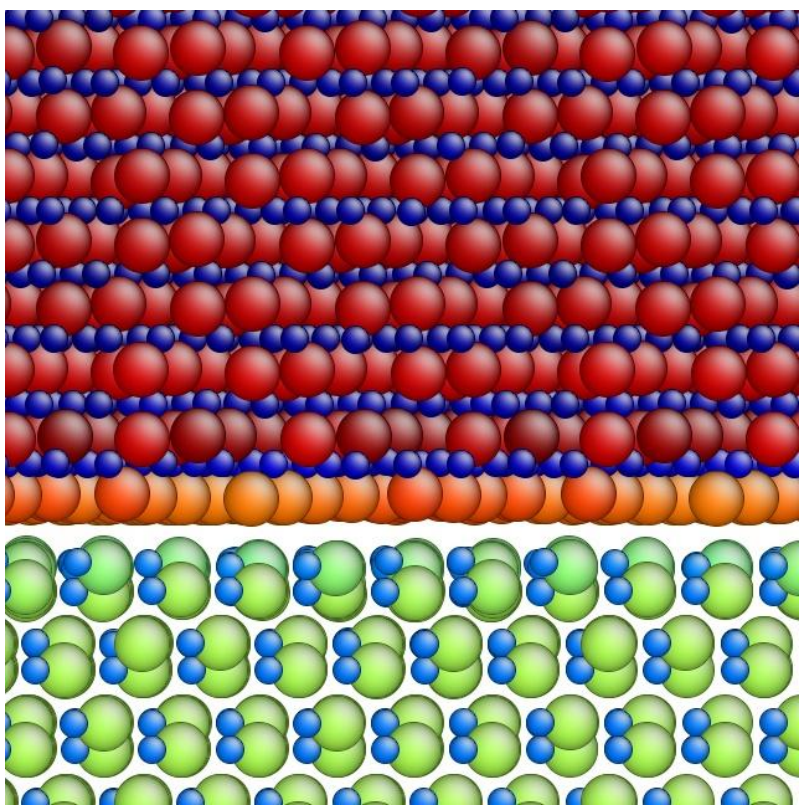

B

Fig. 3 Snapshots of the most stable interface structures for: A) $\operatorname{Al}(111)-\operatorname{AlN}(10 \overline{1} 0)$, B) $\mathrm{Al}_{2} \mathrm{O}_{3}(0001)$-AlN. The atoms are color coded according to charge using the indicated scale.

\subsection{Mechanical Responses of AIN-Nanowires and Nanotubes}

The COMB3 potential was next used to investigate the responses of AlN nanowires and nanotubes to tensile deformation. Nanowire structures were constructed by carving cylinders with diameters of $4.0 \mathrm{~nm}$ and lengths of $10.5 \mathrm{~nm}$ from bulk structures; these dimensions are comparable to the dimensions of samples prepared experimentally by electro-deposition $[4,54]$. Nanotubes of AlN were made by wrapping AlN (100) surface. AlN nanotubes with diameter 4 
$\mathrm{nm}$ and length $14 \mathrm{~nm}$ were considered here. Periodic boundary conditions were applied only in the direction of longest axis of the tubes or wires, which was also the direction along with tensile stress was applied. The nanowire and nanotube consisted of 4352 atoms and 5900 atoms, respectively. The nanostructures were then subjected to a tensile test at a strain rate of $1 \times 10^{8} \mathrm{~s}^{-1}$; such a high rate is typical for MD simulations $[55,56]$. The system was subjected to the NPT ensemble with the Nose-Hoover thermostat and a $0.1 \mathrm{fs}$ time-step. The results of the simulations are illustrated in Fig. 4. The nanowire was predicted to elastically deform up to 0.05 strain and then atomic rearrangement takes place up to the point denoted C. We also predict formation of molecular chains at point D. Such behavior was also observed for other systems, such as for GaN using Tersoff potential [57] and $\mathrm{TiO}_{2}$ nanowires using a Morse empirical potential [58]. 


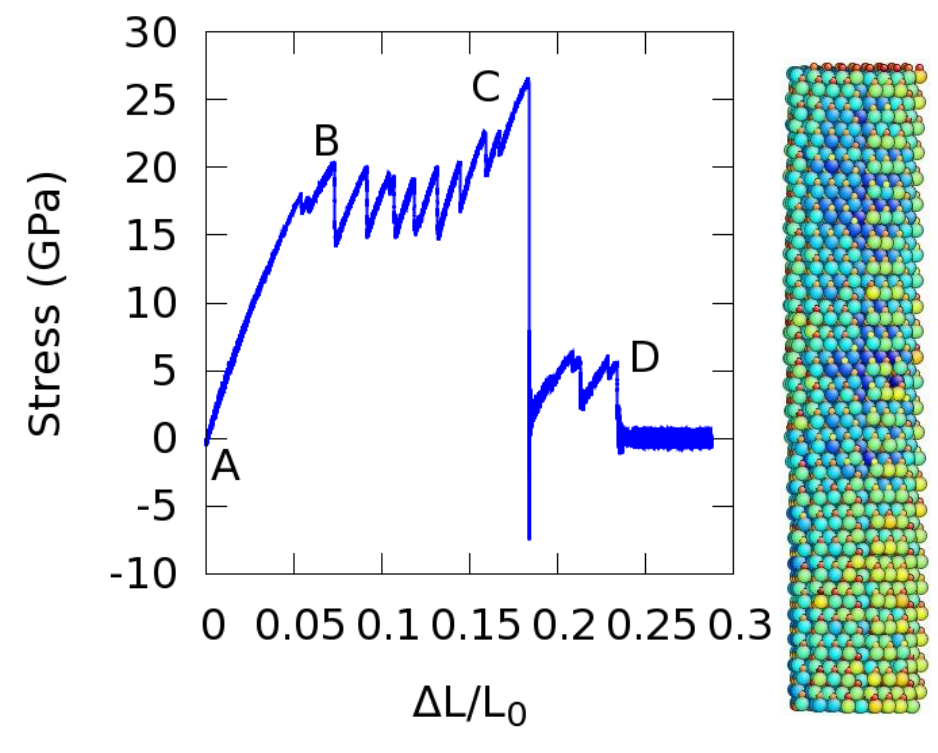

A

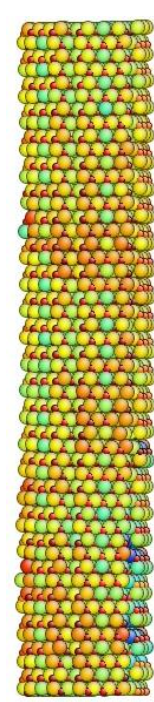

B

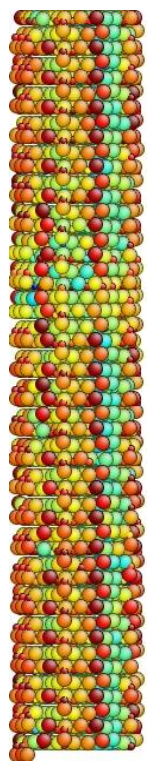

$\mathrm{C}$

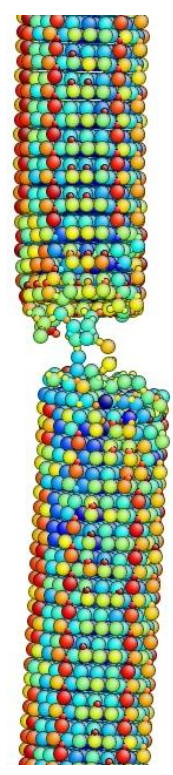

$\mathrm{D}$

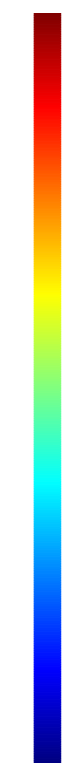

-7.4 bar

Fig. 4 Deformation of AlN nanowire. A molecular chain is predicted to form during the deformation process. The atoms are color coded according to charge using the indicated scale.

The deformation of the AlN nanotube was carried out with similar MD settings. The Young's modulus predicted to be $305 \mathrm{GPa}$ by linear fitting of the stress-strain curve in the linear region of Fig. 5, which was comparable to that of experimental value of 250-400 GPa [2]. The Young's modulus of the nantube is obviously much less than the $\mathrm{C}_{33}$ of bulk AlN. Brittle fracture was predicted to take place with spontaneous notch formation, as illustrated in Fig. 5. Next, we compared the maximum stress $\sigma_{t h}$ and associated strain $\varepsilon_{t h}$ to conventional theory [15] in which these quantities are expressed in terms of elastic constant $E$, surface energy $\gamma$ and interlayer distance $d$ : 


$$
\begin{gathered}
\sigma_{t h}=\sqrt{\frac{E \gamma}{d}} \\
\varepsilon_{t h}=\frac{\pi \sigma_{t h}}{2 E}
\end{gathered}
$$

Based on our empirical potential fitting database and using Eqs. 8 and 9, the calculated $\sigma_{t h}$ and $\varepsilon_{t h}$ for AlN (bulk) was $113 \mathrm{GPa}$ and 0.42 , respectively, while we predicted these values to be $25 \mathrm{GPa}$ and $70 \mathrm{GPa}$, and 0.15 and 0.17 for the AlN nanowire and nanotube, respectively. These differences are attributed to the fact that the stress-strain behavior is determined from MD simulations rather than analytical values, because analytical formulations do not take into account defects that might form during the deformation processes. 


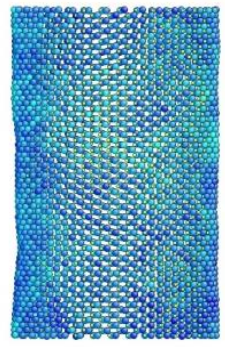

A

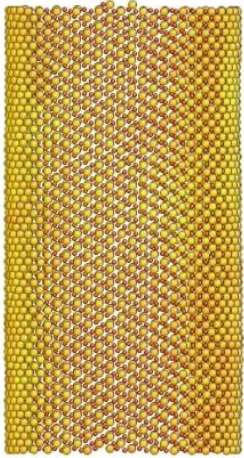

B

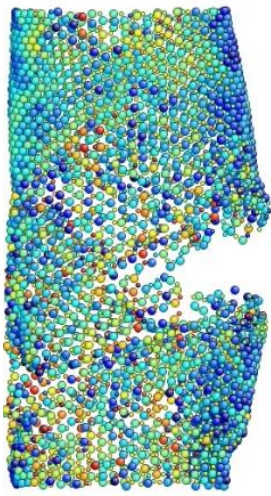

$\mathrm{C}$

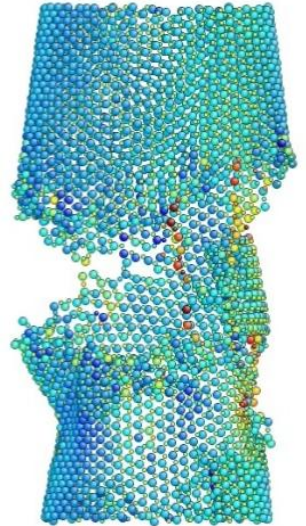

$\mathrm{D}$

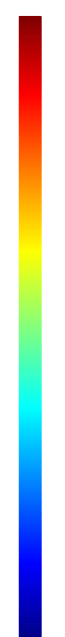

-1.5 bar

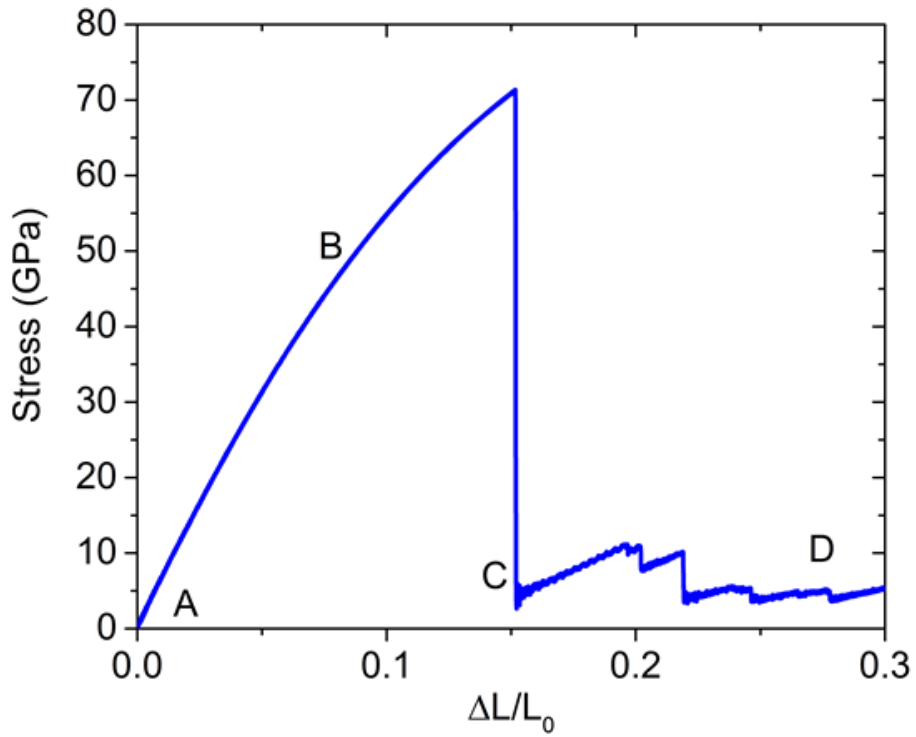

Fig. 5 Deformation behavior of AlN nanotubes. The atoms are color coded according to charge using the indicated scale. 


\section{Conclusions}

A newly developed COMB3 potential was shown to capture the key physical properties of AlN polymorphs; it can also be applied to $\mathrm{Al} / \mathrm{Al}_{2} \mathrm{O}_{3} / \mathrm{AlN}$ interfacial systems and nanostructures. Most importantly, it can be seamlessly coupled with recently developed COMB3 potentials for other materials to enable MD simulation studies of a wide range of heterogeneous material systems. The new potential should therefore prove to be a useful new tool for the computational toolbox and an effective method for carrying out large-scale atomistic simulations of systems of technological importance.

\section{Acknowledgements}

We gratefully acknowledge the support of the U.S. Army Research Office through contract number W911NF-12-1-0153. K.C. acknowledges the support of the Center for Atomic Level Catalyst Design, an Energy Frontier Research Center funded by the U.S. Department of Energy, Office of Science, Office of Basic Energy Sciences under Award Number DE-SC0001058. S.R.P. acknowledges the support of the National Science Foundation through grant number DMR-1005779. 


\section{Appendix:}

The tables below present the COMB3 parameters for AlN. The charge related parameters are given in Table A1 while the bond-order and pairwise parameters are given in Table A2.

Table A1 COMB3 potential charge parameters for AlN.

\begin{tabular}{ccc}
\hline Parameters & $\mathrm{Al}$ & $\mathrm{N}$ \\
\hline $\mathrm{X}\left(\mathrm{eVe}^{-1}\right)$ & 3.10166 & 6.209731 \\
$\mathrm{~J}\left(\mathrm{eVe}^{-1}\right)^{2}$ & 3.53243 & 9.29225 \\
$\mathrm{~K}\left(\mathrm{eVe}^{-1}\right)^{3}$ & 0.48166 & -1.25446 \\
$\mathrm{~L}\left(\mathrm{eVe}^{-1}\right)^{4}$ & 0.0 & 0.286350 \\
$\xi\left(\overline{\mathrm{A}}^{-1}\right)$ & 2.1433 & 1.438711 \\
$\mathrm{Z}(\mathrm{e})$ & 0.052709 & -0.552136 \\
$\mathrm{P}^{x}\left(\mathrm{eV} \cdot \mathrm{e}^{-3} \cdot \mathrm{r}^{-3}\right)$ & 1.5063 & 3.7385490 \\
$\mathrm{P}^{\mathrm{J}}\left(\mathrm{eV} \cdot \mathrm{e}^{-3} \cdot \mathrm{r}^{-3}\right)$ & 0.2 & 0.5167863 \\
$\mathrm{D}_{\mathrm{U}}(\stackrel{\overline{\mathrm{A}}}{)}$ & -0.03512360 & 0.5167863 \\
$\mathrm{D}_{\mathrm{L}}(\stackrel{\mathrm{A}}{)})$ & 0.0105133 & 0.1969380 \\
$\mathrm{Q}_{\mathrm{U}}(\mathrm{e})$ & 3.00000000 & 5.000000 \\
$\mathrm{Q}_{\mathrm{L}}(\mathrm{e})$ & -5.000 & -3.000000 \\
\hline
\end{tabular}


Table A2 COMB3 pairwise and bond-order parameters for AlN

\begin{tabular}{|c|c|c|c|c|}
\hline Parameters & $\mathrm{Al}-\mathrm{Al}$ & Al-N & $\mathrm{N}-\mathrm{Al}$ & $\mathrm{N}-\mathrm{N}$ \\
\hline$A_{i j}(e V)$ & 473.3379 & 1677.4790 & 1677.4790 & 7654.97300 \\
\hline$B_{i j}^{1}(e V)$ & 242.8881 & 241.88890 & 241.88890 & 2102.2960 \\
\hline$B_{i j}^{2}(e V)$ & 0.0000 & 0.0 & 0.0 & 0.0 \\
\hline$B_{i j}^{3}(e V)$ & 0.0000 & 0.0 & 0.0 & 0.0 \\
\hline$\lambda\left(\AA^{-1}\right)$ & 1.8617 & 3.56577 & 3.56577 & 5.21803 \\
\hline$\alpha_{i j\left(\AA^{-1}\right)}^{1}$ & 1.4993 & 1.81353 & 1.81353 & 3.73854 \\
\hline$\alpha_{i j}^{2}\left(\AA^{-1}\right)$ & 1.1 & 0.0 & 0.0 & 0.0 \\
\hline$\alpha_{i j}^{3}\left(\AA^{-1}\right)$ & 0.6858 & 0.0 & 0.0 & 0.0 \\
\hline$\beta_{\left(\AA^{-1}\right)}$ & 1.4993 & 1.16517 & 0.0 & 3.738549 \\
\hline $\mathrm{b}_{6}$ & 0.0000 & 0.0 & 0.0 & -1.0257 \\
\hline$b_{5}$ & 0.0000 & 0.0 & 0.0 & -0.1700 \\
\hline $\mathrm{b}_{4}$ & 0.0000 & 0.0 & 0.0 & 2.0153 \\
\hline$b_{3}$ & 0.0000 & 0.0 & 0.0 & -2.2384 \\
\hline $\mathrm{b}_{2}$ & 0.0000 & 0.0 & 0.0 & 1.1852 \\
\hline$b_{1}$ & 0.0000 & 0.0 & 0.0 & 2.7092 \\
\hline $\mathrm{b}_{0}$ & 0.0327 & 0.074639 & 1.5931860 & 1.6913250 \\
\hline $\mathrm{R}(\AA)$ & 3.4 & 2.56 & 2.56 & 2.0 \\
\hline $\mathrm{S}(\AA ̊)$ & 3.7 & 2.96 & 2.96 & 2.3 \\
\hline $\begin{array}{l}\mathrm{LP}_{0} \\
\mathrm{LP}_{1}\end{array}$ & $\begin{array}{c}0.0 \\
-0.00687\end{array}$ & $\begin{array}{c}-0.03 \\
1.1\end{array}$ & $\begin{array}{l}0.0 \\
0.0\end{array}$ & $\begin{array}{l}0.0 \\
0.0\end{array}$ \\
\hline $\mathrm{LP}_{2}$ & -0.00446 & 1.0 & 0.0 & 0.0 \\
\hline $\mathrm{LP}_{3}$ & -0.04001 & 0.0 & 0.0 & 0.0 \\
\hline $\mathrm{LP}_{4}$ & -0.01405 & 0.16 & 0.2 & 0.0 \\
\hline $\mathrm{LP}_{5}$ & 0.003549 & 0.0 & 1.1 & 0.0 \\
\hline
\end{tabular}




\section{References:}

[1] B. D. Bloor, R.W. Kahn, Encyclopedia of Advanced Materials Pergamon press, Cambridge, 1994.

[2] G. Stan, C. Ciobanu, T. Thayer, G. Wang, J. Creighton, K. Purushotham, L. Bendersky, R. Cook, Nanotech., 20 (2009) 035706.

[3] H.-C. Hsu, G.-M. Hsu, Y.-s. Lai, Z.C. Feng, S.-Y. Tseng, A. Lundskog, U. Forsberg, E. Janzén, K.-H. Chen, L.-C. Chen, App. Phys. Lett., 101 (2012) 121902.

[4] S. Zhao, A. Connie, M. Dastjerdi, X. Kong, Q. Wang, M. Djavid, S. Sadaf, X. Liu, I. Shih, H. Guo, Scientific reports, 5 (2015).

[5] Y.-R. Lin, S.-T. Wu, Surface science, 516 (2002) L535-L539.

[6] C.M. Montesa, N. Shibata, T. Tohei, Y. Ikuhara, J. Mat. Sci., 46 (2011) 4392-4396.

[7] Y. Tokumoto, Y. Sato, T. Yamamoto, N. Shibata, Y. Ikuhara, J. Mat. Sci., 41 (2006) 25532557.

[8] S. Strite, H. Morkoç, J. Vac. Sci. \& Tech. B, 10 (1992) 1237-1266.

[9] R. Vispute, H. Wu, J. Narayan, App. Phys. Lett., 67 (1995) 1549-1551.

[10] R. Cremer, M. Witthaut, K. Reichert, M. Schierling, D. Neuschütz, Surface and Coatings Technology, 108 (1998) 48-58.

[11] J. Hafner, C. Wolverton, G. Ceder, MRS bulletin, 31 (2006) 659-668.

[12] E. Wimmer, Journal of Computer-Aided Materials Design, 1 (1994) 215-242.

[13] R. Di Felice, J.E. Northrup, App. Phys. Lett., 73 (1998) 936-938.

[14] S. Ogata, H. Kitagawa, Comp. Mater. Sci., 15 (1999) 435-440.

[15] A. Griffith, Philosophical transactions of the royal society of london. Series A, , (1921) 163198.

[16] Y. Li, Z. Zhou, P. Shen, S. Zhang, Z. Chen, Nanotech., 20 (2009) 215701.

[17] J. Chisholm, D. Lewis, P. Bristowe, J. Phys.: Cond. Mat., 11 (1999) L235.

[18] M. Tungare, Y. Shi, N. Tripathi, P. Suvarna, F.S. Shahedipour-Sandvik, Physica Status Solidi (A), 208 (2011) 1569-1572.

[19] F. Benkabou, H. Aourag, P.J. Becker, M. Certier, Molecular Simulation, 23 (2000) 327-341.

[20] D. Powell, M. Migliorato, A. Cullis, Phys. Rev. B, 75 (2007) 115202.

[21] J. Kioseoglou, P. Komninou, T. Karakostas, Physica Status Solidi (B), 245 (2008) 11181124.

[22] J.W. Kang, H.J. Hwang, Comput. Mater. Sci. , 31 (2004) 237-246.

[23] P. Vashishta, R.K. Kalia, A. Nakano, J.P. Rino, J. App. Phys. , 109 (2011) 033514.

[24] K. Choudhary, T. Liang, A. Chernatynskiy, Z. Lu, A. Goyal, S.R. Phillpot, S.B. Sinnott, J. Phys.: Cond. Mat. , 27 (2015) 015003.

[25] K. Choudhary, T. Liang, A. Chernatynskiy, S.R. Phillpot, S.B. Sinnott, J. Phys. : Cond. Matt., 27 (2015) 305004.

[26] J.Martinez ,T.Liang., S.B.Sinnott,S.R.Phillpot, J. Phys Chem A, (in prep).

[27] B. Devine, T.R. Shan, Y.T. Cheng, A.J.H. McGaughey, M. Lee, S.R. Phillpot, S.B. Sinnott, Phys. Rev. B, 85 (2012).

[28] M.J. Noordhoek, T. Liang, Z.Z. Lu, T.R. Shan, S.B. Sinnott, S.R. Phillpot, J. Nucl. Mater., 441 (2013) 274-279.

[29] Y.-T. Cheng, T.-R. Shan, T. Liang, R.K. Behera, S.R. Phillpot, S.B. Sinnott, J. Phys. : Cond. Mat., 26 (2014) 315007. 
[30] M. J. Noordhoek, T. Liang T- Chiang, S. B. Sinnott, S. R. Phillpot, J. Nuc. Mater. 452 (2014) 285.

[31] Y.-T. Cheng, T.-R. Shan, T. Liang, R.K. Behera, S.R. Phillpot, S.B. Sinnott, J. Phys.: Cond. Matt., 26 (2014) 315007.

[32] T. Liang, T.-R. Shan, Y.-T. Cheng, B.D. Devine, M. Noordhoek, Y. Li, Z. Lu, S.R. Phillpot, S.B. Sinnott, Materials Science and Engineering: R: Reports, 74 (2013) 255-279.

[33] G. Kresse, J. Hafner, Phys. Rev. B, 47 (1993) 558-561.

[34] G. Kresse, J. Hafner, Phys. Rev. B, 49 (1994) 14251-14269.

[35] G. Kresse, J. Furthmüller, Comput. Mater. Sci. , 6 (1996) 15-50.

[36] G. Kresse, J. Furthmüller, Phys. Rev. B, 54 (1996) 11169-11186.

[37] J.A. Martinez, D.E. Yilmaz, T. Liang, S.B. Sinnott, S.R. Phillpot, Current Opinion in Solid State and Materials Science, 17 (2013) 263-270.

[38] C.R. Rao, A. Fieger, C. Heumann, T. Nittner and S. Scheid Springer Series in Statistics, Berlin (1999).

[39] S. Plimpton, J. Comput. Phys., 117 (1995) 1-19.

[40] A. Zoroddu, F. Bernardini, P. Ruggerone, V. Fiorentini, Phys. Rev. B, 64 (2001) 045208.

[41] E. Ruiz, S. Alvarez, P. Alemany, Phys. Rev. B, 49 (1994) 7115.

[42] A. Hung, S.P. Russo, D.G. McCulloch, S. Prawer, J. Chem. Phys., 120 (2004) 4890-4896.

[43] D. Holec, P.H. Mayrhofer, Scripta materialia, 67 (2012) 760-762.

[44] R. Mishra, O.D. Restrepo, S. Rajan, W. Windl, App. Phys. Lett., 98 (2011) 232114.

[45] W.W. Tipton, R.G. Hennig, J. Phys.: Cond. Matt., 25 (2013) 495401.

[46] W.W. Tipton, C.R. Bealing, K. Mathew, R.G. Hennig, Phys. Rev. B, 87 (2013) 184114.

[47] A. Jain, S.P. Ong, G. Hautier, W. Chen, W.D. Richards, S. Dacek, S. Cholia, D. Gunter, D. Skinner, G. Ceder, APL Materials, 1 (2013) 011002.

[48] M.M. Islam, A. Ostadhossein, O. Borodin, A.T. Yeates, W.W. Tipton, R.G. Hennig, N. Kumar, A.C. van Duin, Physical Chemistry Chemical Physics, 17 (2015) 3383-3393.

[49] M. Schwoerer-Böhning, A. Macrander, M. Pabst, P. Pavone, Physica Status Solidi B Basic Research, 215 (1999) 177-180.

[50] J. Nipko, C.-K. Loong, Phys. Rev. B, 57 (1998) 10550.

[51] A. Chernatynskiy, S.R. Phillpot, Computer Physics Communications, 192 (2015) 196-204.

[52] R. Couturier, D. Ducret, P. Merle, J. Disson, P. Joubert, J. Euro. Ceram. Soc., 17 (1997) 1861-1866.

[53] A. Zur, T. McGill, J. App. Phys., 55 (1984) 378-386.

[54] M. Meyyappan, M.K. Sunkara, Inorganic nanowires: applications, properties, and characterization, CRC Press, 2009.

[55] M.R. Sørensen, M. Brandbyge, K.W. Jacobsen, Physical Review B, 57 (1998) 3283.

[56] L. Pastor-Abia, M. Caturla, E. SanFabian, G. Chiappe, E. Louis, Phys. Rev. B, 83 (2011) 165441.

[57] Y.-R. Jeng, P.-C. Tsai, T.-H. Fang, Nanotech., 15 (2004) 1737.

[58] L. Dai, C. Sow, C. Lim, W. Cheong, V. Tan, Nano letters, 9 (2009) 576-582. 